Case Report

\title{
Medial-Inguinal Approach: A Novelty in Hip Joint Replacement
}

\author{
Luca Lucente*, Andrea Palmesi and Damiano Longo \\ Nuova Itor Hospital, Rome, Italy
}

\begin{abstract}
Introduction: True to the concept of Tissue Sparing Surgery, we developed this new surgical technique to reach the coxofemoral joint by starting at the inguinal-medial region.

Methods: We performed total hip arthroplasty on 150 patients suffering from hip arthritis.

Results: In our study, operation time and blood loss were lower, there were no complications, and recovery time was shorter.

Discussion: We have developed a surgical process that allows for safe, easy and fast replacement of the hip that spares the hip stabilizer muscles completely. Throughout the operation, the surgeon can view the acetabulum from the front, which is a view that is preferable to the one available with other known techniques. There is no need for special equipment or special operating tables, and surgeons do not face a steep learning curve when first introduced to the procedure. Since risks of dislocation are non-existent, the patient is allowed to lie in bed in any position. The procedure is preferable aesthetically since any scarring is hidden from view in the inguinal folds of skin. Patients can resume walking immediately, using two Canadian crutches for only a few days.

Conclusion: The authors believe, thanks to its low cost and ease of performance and replication, this technique offers nothing but advantages for the patient. Easier rehabilitation is another positive aspect. The procedure can be considered a valid alternative to other common surgical approaches.
\end{abstract}

\section{Keywords}

The medial-inguinal approach, A new surgical approach to the hip, Innovation in hip surgery

\section{Introduction}

Reaching the hip joint via the medial region is not a novelty. In 1908, Ludloff hypothesized the procedure as a way to reduce congenital hip dislocation. In an article published in 1913, Ludloff described gaining access through the medial region as a simple, fast, and safe way to carry out tenotomy of the iliopsoas muscle that blocked the bloodless reduction of the femur head in the acetabulum [1,2]. In later years, several authors took an interest in the procedure, and they each contributed certain modifications: Ferguson in 1973 [3]. All these authors used this technique only in newborn patients for the sole purpose of performing iliopsoas tenotomy [4].

Since this technique is used in newborns, it must be a simple, fast, and non-invasive surgical procedure, with low risks of perioperative complications. Hence, we devised the idea to go through the medial-inguinal area to implant a hip prosthetic.

\section{Materials and Methods}

We implanted 150 full hip prostheses on patients suffering from hip arthritis. The majority of the patients were female (88 female patients, 62 male patients). The average age of patients undergoing surgery for a full hip replacement was 65, with a Harris average equal to 65 . Female patients underwent vaginal disinfection 3 days before surgery, the morning of surgery, and three days after surgery with chlorhexidine or $10 \%$ betadine-based products. One hour before operating time, all patients underwent antibiotic prophylaxis and, unless otherwise noted, an intravenous inoculation of $1 \mathrm{gr}$. of tranexamic acid. Before sterilizing the operating field, all patients were scrubbed around the area of incision with a chlorhexidine or betadine-based solution. We never had to use draining because blood loss was so low.

Post-operation, we did not use devices to maintain the lower limbs spread or toilet seat risers. Since the risk of dislocation was close to zero, patients were able to lie

*Corresponding author: Luca Lucente, Nuova Itor Hospital, Via Montaione 40 - 00139 Rome, Italy

Accepted: March 06, 2019

Published online: March 08, 2019

Citation: Lucente L, Palmesi A, Longo D (2019) Medial-Inguinal Approach: A Novelty in Hip Joint Replacement. J Orthop Surg Tech 2(1):62-67

Copyright: (C) 2019 Lucente L, et al. This is an open-access article distributed under the terms of the Creative Commons Attribution License, which permits unrestricted use, distribution, and reproduction in any medium, provided the original author and source are credited. 
down in their preferred position right away, as long as the chosen position was not too extreme. With the exception

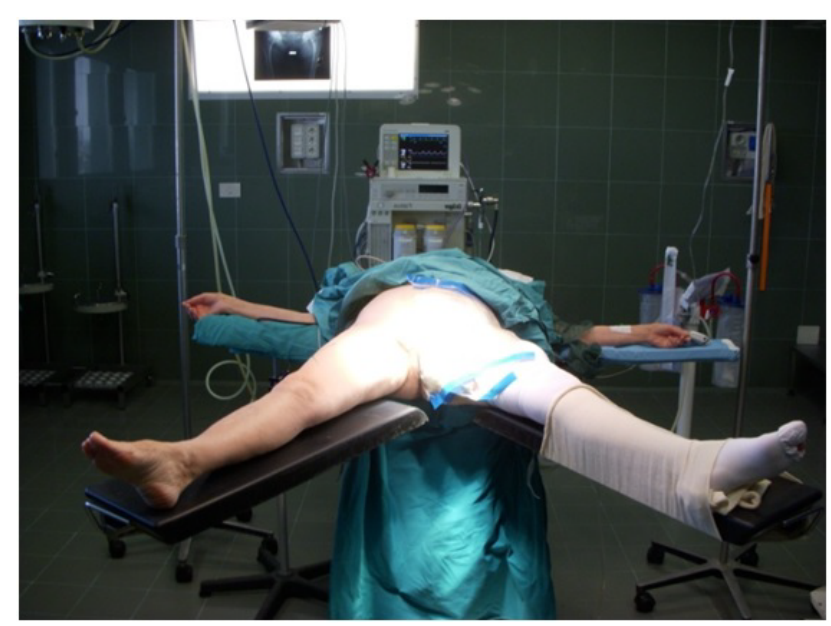

Figure 1a: Patient's position on a standard operating table.

\section{CEILING VIEW OF OPERATING THEATER}

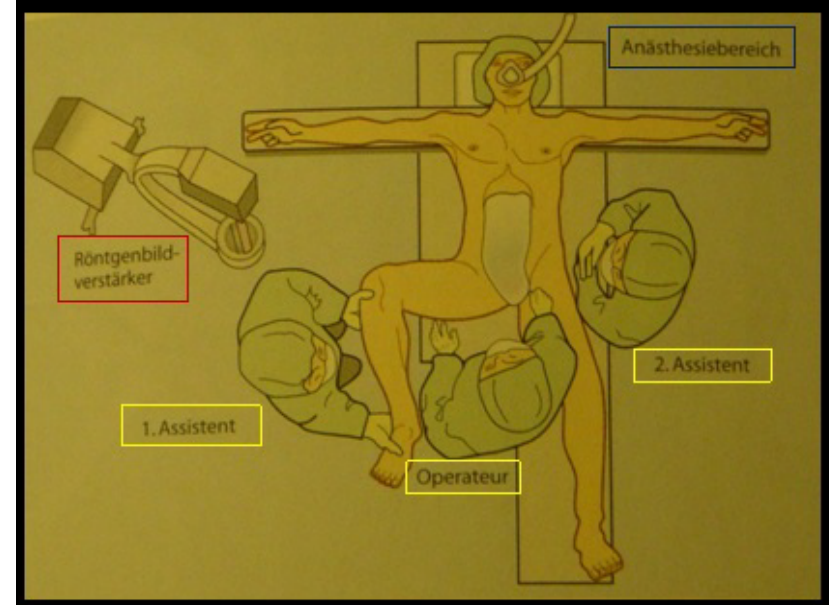

Figure 1b: The operating room. of comorbidity cases, which mostly afflicted more elderly patients with femoral neck fractures, all other patients were able to walk a few hours after surgery. All patients went through a brief rehabilitation programme. They were evaluated using the Harris Hip Score at 1, 3, and 6 months and 1 year after surgery.

\section{Surgical Technique}

Patients were placed on their back on a standard operating table for lower limb abduction and hyperextension of the limb on which to operate. The waist was shifted so that the side requiring the operation lay next to the external edge. Articulated supports were then placed on the operating table to ensure the waist was perfectly aligned and could not move at all. The lower limbs were abducted (Figure 1a), so that the surgeon could sit between them. The first assistant was positioned on the same side that required the surgery. The second assistant flanked the side that did not require surgery (Figure 1b). After having adequately prepped the operative field, the limb requiring the operation was flexed and abducted in the "frog leg" position. A cutaneous incision approximately $8 \mathrm{~cm}$ in length was curved and centered on the cutaneous projection of the adductor longus tendon, which was approximately $5 \mathrm{~cm}$ from the inguinal fold (Figure 1c). The subcutaneous tissue was cut in order to reach the adductor longus tendon. The intermuscular plane is exploited among the muscle's adductor longus and pectineus. Retracting the pectineus muscle with a curved Hohmann retractor allowed for access to the hip articular capsule. The pectineus muscle constituted the bottom part of the triangle of Scarpa and retracting it afforded protection of the femoral vascular nerve fascia. Before proceeding with the capsulotomy, the medial circumflex branch of the femoral artery was isolated, ligated, and sectioned. Prior to optional luxation of the femoral head, we proceeded to the capsulotomy and the successive osteotomy of the femoral neck. Once it was exposed with Hohmann retractors, the surgeon had a complete frontal view of the acetabulum circumference (Figure 2a). We continued with the preparation of the acetabulum with
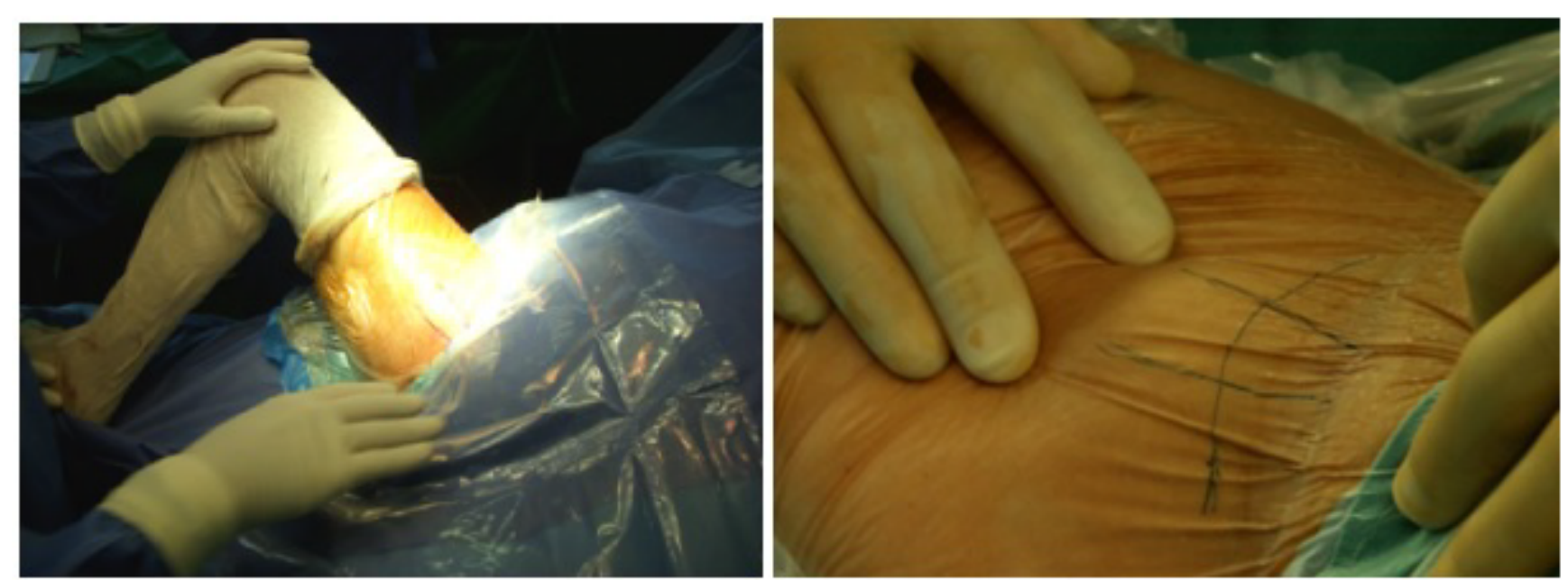

Figure 1c: The limb to be operated is in "frog leg position". The cutaneous incision is about $8 \mathrm{~cm}$ long and is curved and centered on the cutaneous projection of the adductor longus tendon, which is about $5 \mathrm{~cm}$ from the inguinal fold. 


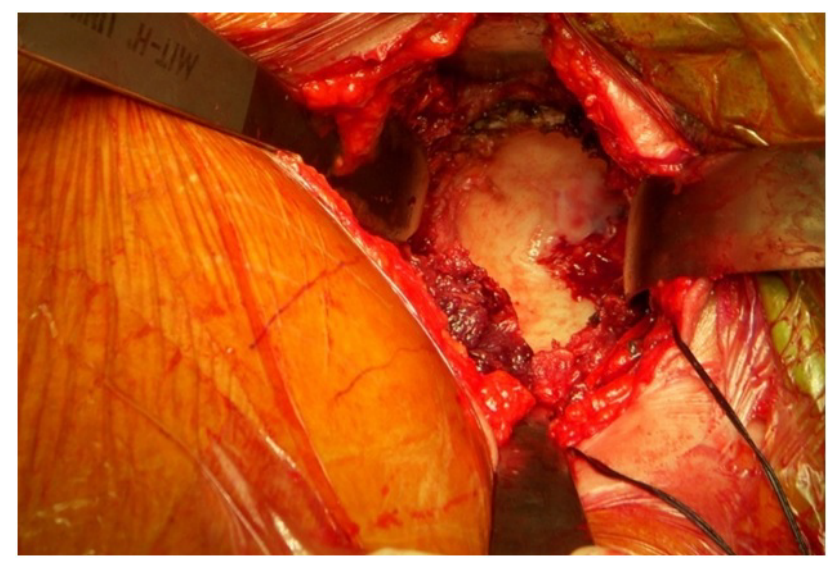

Figure 2a: Complete frontal view of the acetabulum circumference. standard acetabular frese, and we positioned the acetabulum and the test insert (Figure $2 b$ ). The correct positioning of the cup is extremely easy since the acetabulum is visible in its whole circumference. With the handle of the cup parallel to the floor we have an anteversion equal to $0^{\circ}$. To increase the degrees of antiversion the handle of the cup must be lifted up to reach the wanted degrees $\left(15^{\circ}-20^{\circ}\right)$. To get a correct side inclination of the cup needs to abduct the handle of the cup up to reach the wanted degrees $\left(35^{\circ}-45^{\circ}\right)$, considering $0^{\circ}$ of side inclination when the handle of the cup is parallel to the axis of the femur placed in neutral position. To prepare the femoral canal, we hyperextended the femur by lowering the operating table's lower limb support base approximately 30 degrees. Then, with the aid of a hook inserted into the femoral canal and with a distalizing manoeuvre, we shifted
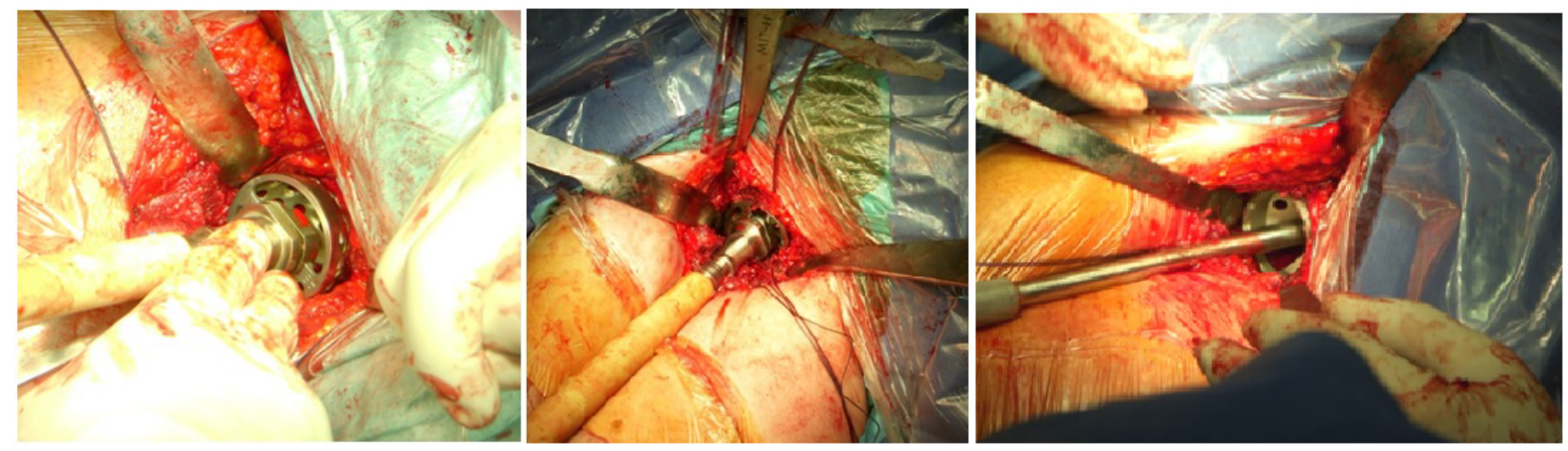

Figure $\mathbf{2 b}$ : Preparing of the acetabulum with standard acetabular frese and positioning the acetabulum and the test insert.

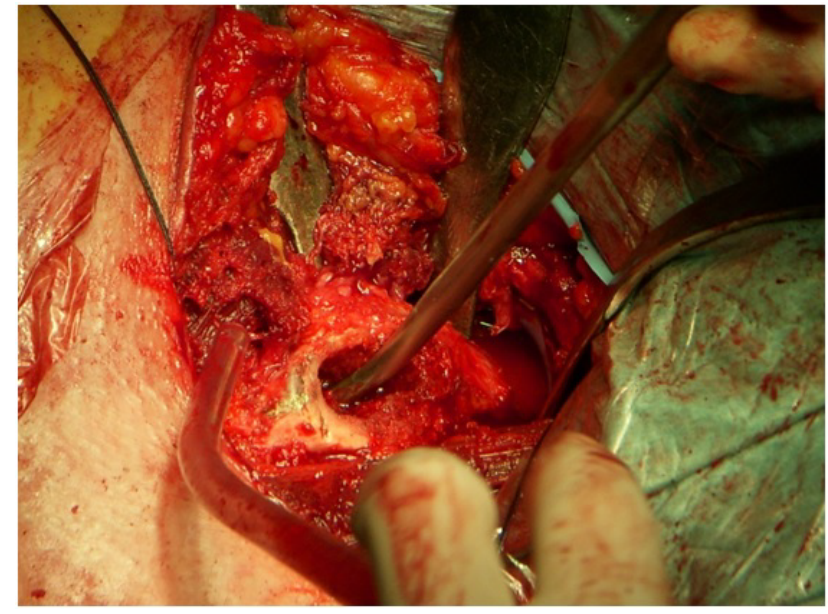

Figure 3a: With the aid of a hook inserted into the femoral canal and with a distalizing manoeuvre, we shifted the greater trochanter from the acetabular border.

the greater trochanter from the acetabular border (Figure 3a). At this point, the lower limb was moved from the operating table support base to a sterile sack previously prepared with canvases during the setup of the operating field (Figure 3b). By this point, the femoral canal was widely exposed, and the positioning of the test femoral stem and head could be prepped with ease (Figure $3 c$ ). As for the cup

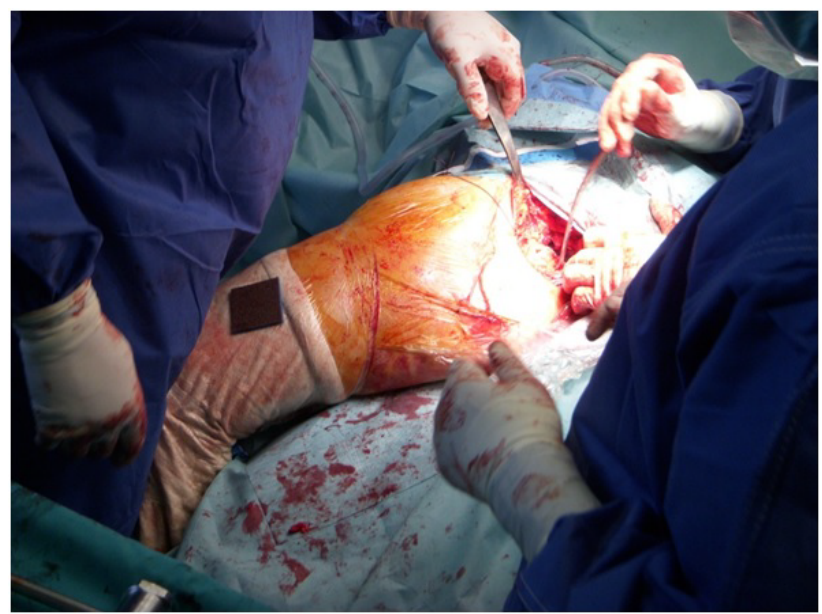

Figure 3b: The lower limb was moved from the operating table support base to a sterile sack.

the positioning of the prosthetic stem is extremely easy thanks to the good vision of the femoral canal and the less trocanther. It's very important to work the femoral canal to the great trocanther to avoid to install the stem in varus position. We reduced the prosthesis and its test components; we raised the operating table's lower limb support base to the same height of the counter-lateral support base; and we placed both legs in neutral position to monitor metrics 

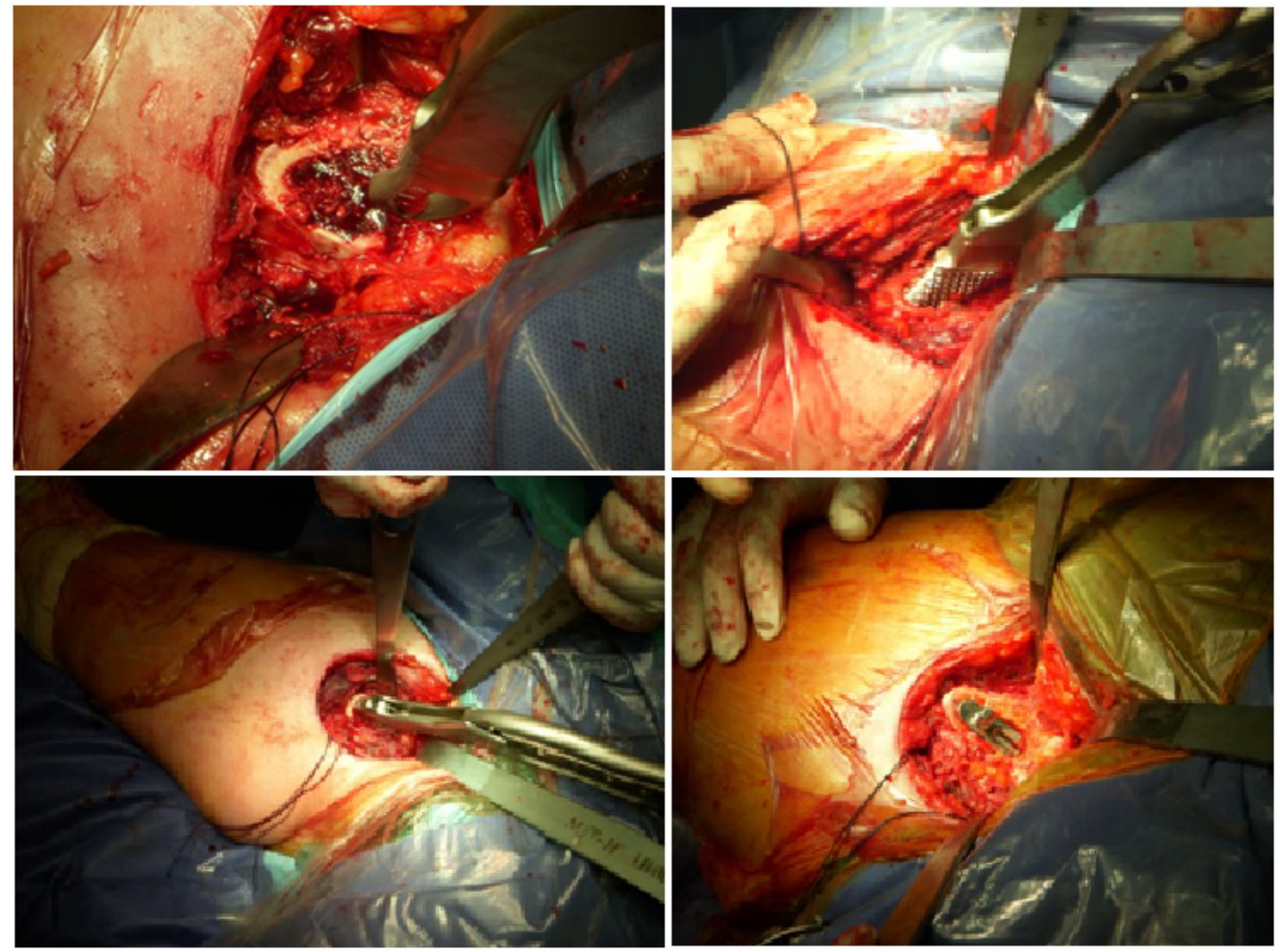

Figure 3c: Preparing the femoral canal and setting the test femoral stem.
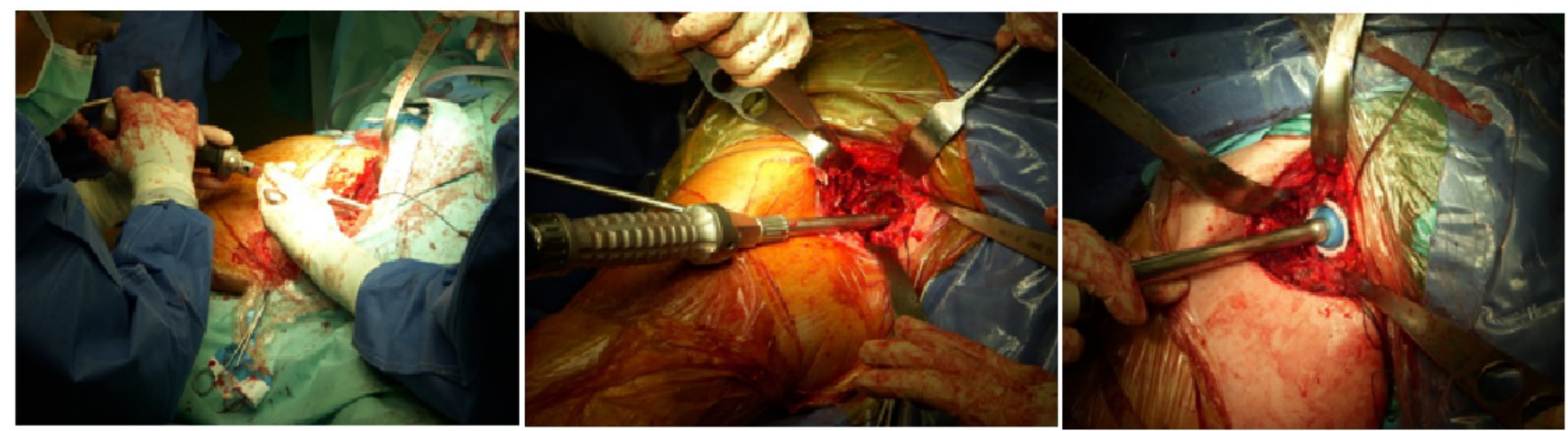

Figure 3d: Positioning of definitive prosthetic acetabulum.
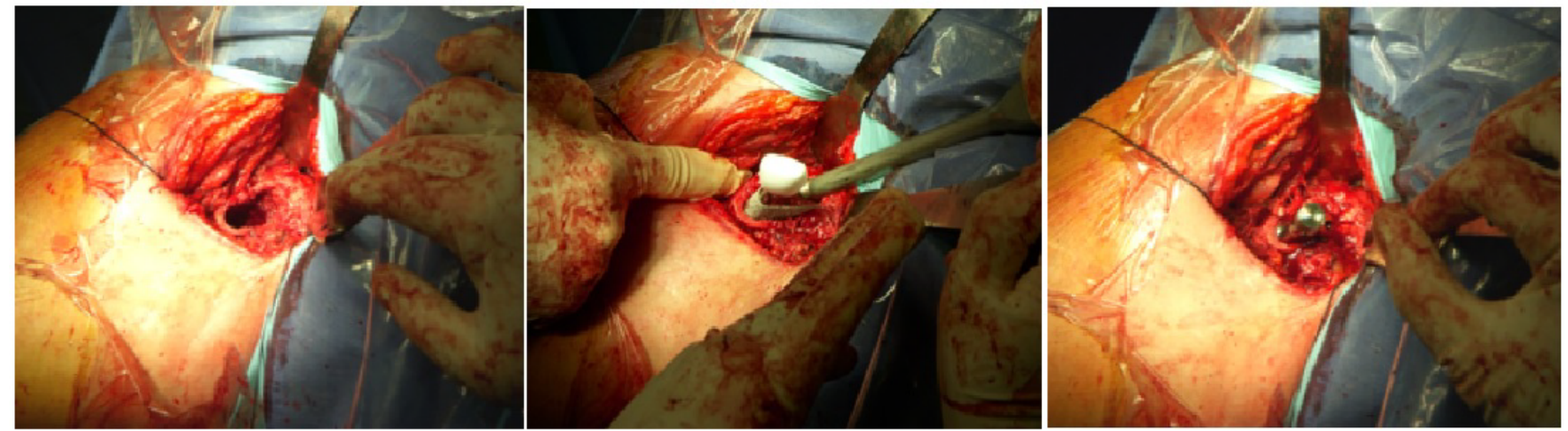

Figure 3e: Positioning of definitive prosthetic stem.

and perform all the movements needed to measure the functionality and stability of the prosthetic implant. Once these trials were completed, we removed the test parts and implanted the actual prosthetic by following the same steps 
as above (Figure $3 d$, Figure 3e, Figure $3 f$ and Figure $4 a)$. We always use highly cross-linked polyethylene articulating with ceramic or ceramic-on-ceramic as bearing combination. We never use metal-on-metal bearing combination. If the capsule was preserved, we proceeded to perform capsulorrhaphy; if not, we proceeded directly to suturing first the subcutaneous, then the cutaneous, plane. All that was required was a light compressive dressing. Before being brought back to recovery, the patient underwent a standing X-ray exam of the operated hip (Figure 4b).

\section{Results}

We obtained operational times of 60 minutes, with a minimum of 45 minutes and a maximum of 90 minutes. Obviously, times became shorter as we went along the learning curve. Blood loss was extremely low, 200 cc. average, and such that there was no need for a transfusion. We encountered no prosthesis dislocations, aseptic or septic mobilization of the prosthetic implant, or vascular and/or nerve damage. Moreover, we observed no ossification or thromboembolic events. In one case, a patient developed a lymphangitis of the operated limb, but it was treated pharmacologically. There was only one case of delayed

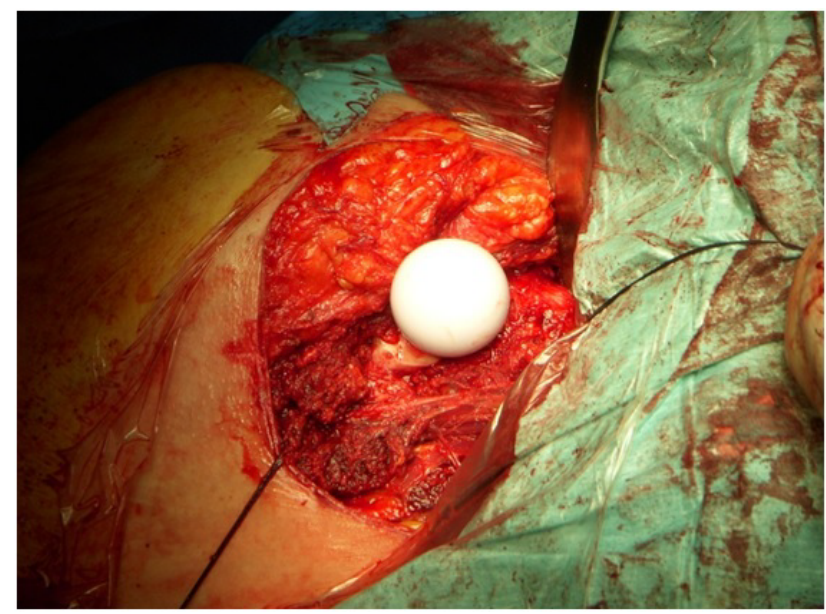

Figure 3f: Positioning of definitive prosthetic head.

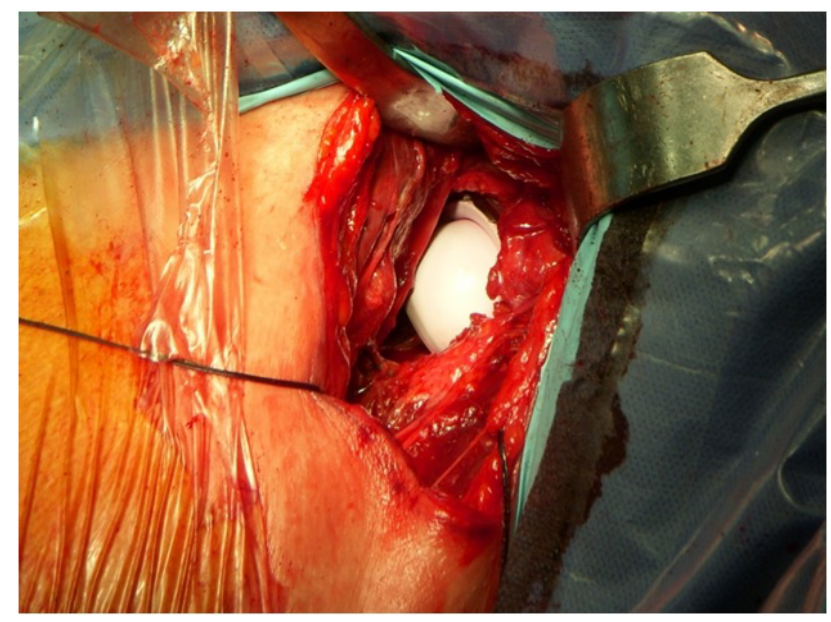

Figure 4a: View of the reduced prosthesis. healing of the surgical wound due to a superficial infection treated with surgical toilette and prescribed antibiotics. All patients except those with a comorbidity that delayed a speedy recovery, were able to walk a few hours after surgery. Two days after the operation, they were able to move autonomously with or without Canadian crutches, depending on their level of compliance. Thirty days after surgery, and with the most motivated and collaborating patients, we obtained an average score of 93.7 with a standard deviation of \pm 8.2 in the Harris Hip Score. The Harris Hip Score was then evaluated at 3 and 6 months and 1 year after surgery. At 3 months, the Harris Hip Score was 95.8 with a standard deviation of \pm 6 .9. At 6 months, the Harris Hip Score reached a value of 96.8 with a standard deviation of \pm 6.1 . At 1 year, the Harris Hip Score was 98.8 with a standard deviation \pm 4.8 .

\section{Discussion}

By combining the concept of Tissue Sparing Surgery with the need for an easy, safe, and fast procedure, we began studying a new surgical approach that provides the most direct way possible to the hip joint. We began by referencing Ludloff's studies from the early 1900s. He proposed a surgical procedure that would reach the hip through the inguinalmedial area. His technique, which has undergone changes over the years, is still the most widely used today to reduce the femoral head in the acetabular cavity in newborn patients who suffer from congenital hip dislocation. This technique has been proven to be conservative, risk-free, easily carried out and feasible in short operating times [3-5]. In the early 2000s, after taking such characteristics into account, together

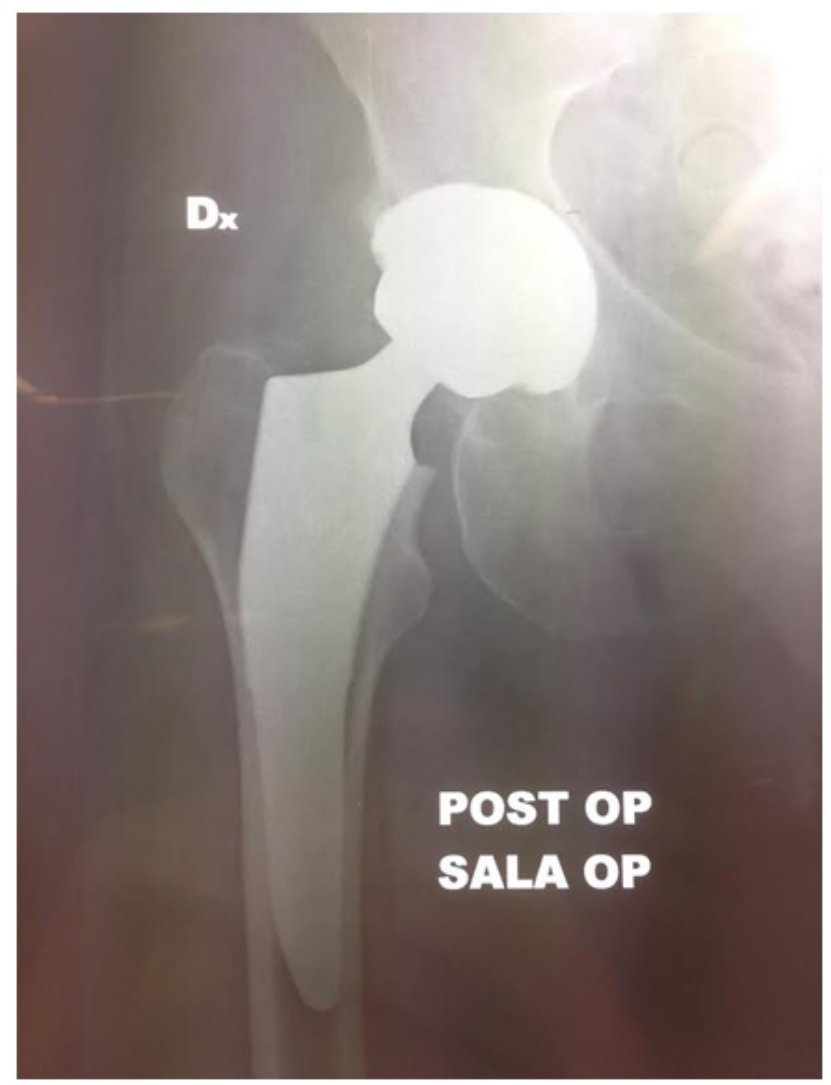

Figure 4b: X-ray post-op control. 


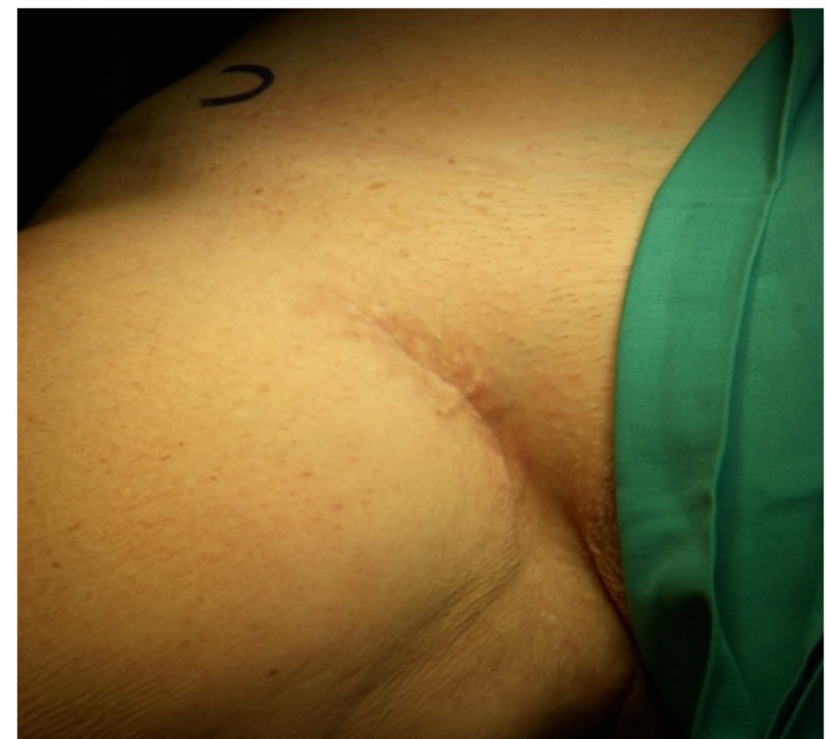

Figure 5: View of the surgical scar.

with Prof. Wolfram, we started looking for a new surgical path to implant hip prostheses [6-9]. We abstained from this technique, however, because the preparation of the femoral canal and the subsequent implant of a femoral stem were particularly difficult. Following numerous anatomical studies in cadaver labs, we made the necessary changes to the procedure in order to make it appropriate for implanting hip prostheses. It is truly a Tissue Sparing Surgery, because no muscle or tendon is sacrificed. It is an extremely safe technique because the medial circumflex femoral artery is the only anatomical structure that we need to watch out for and this is done first, by ligating it and then sectioning it. For our purposes, this is irrelevant, since the artery supplies blood exclusively to the femoral head. We divaricate the pectineus muscle and then arrive at the articular capsule in less time than other known surgeries. Even the closing of the operational site is much quicker, because we only had the subcutaneous and cutaneous levels to suture.

The surgeon has a better view of the acetabulum because he or she can look at its entire circumference straight on. This allows for an easy preparation of the acetabulum and avoids poor positioning of the prostheses. The same goes for the femur. In fact, we never needed X-rays during operations.

This is a versatile procedure that, thanks to the excellent surgical view, allows surgeons to implant easily the prostheses in correct position. The procedure's only contraindication is ankylosis, and we advise against resorting to it with patients who have a $\mathrm{BMI}$ value $\geq 32$.

Managing patients in the ward is simple. Immediately after surgery, patients can lie in their preferred position, as long as it is not extreme. They do not need lower limb spreading devices, nor do they need toilet seat risers, and genital hygiene is particularly easy.

The hip stabilizing muscles are spared and the patients sense improved stability right away. For this reason, they use Canadian crutches for a short time, and their rehabilitation is easy and short. With this technique, surgeons can implant all types of hip prosthetics commercially available, contributing to considerable savings for the prosthetics industry. From a surgical point of view, it is an easy technique that is easily replicated with a short learning curve. The last advantage is an aesthetic one, particularly appreciated by young, female patients, because the scar is approximately $8 \mathrm{~cm}$ and is practically invisible since it is hidden between inguinal skin folds (Figure 5).

\section{Conclusion}

The authors believe that the inguinal-medial approach is a perfect example of Tissue Sparing Surgery. Because of its lack of complications inside and outside the operating room and because of the reduced hospital and recovery time for patients, the procedure lowers the social costs of hip replacement surgery. Always in the concept of tissue sparing surgery, patients operated with this technique, not having suffered damage to the muscles which stabilize the hip, will be able to deal with a possible revision surgery with considerably better results than those who are subjected to a first prosthetic implant through a lateral or posterolateral access. While not being a replacement for other existing techniques, this procedure is an extremely advantageous alternative for surgeons and especially for younger patients.

\section{References}

1. K Ludloff (1913) The open reduction of the congenital hip dislocation by an anterior incision. Am J Orthop Surg 10: 438454.

2. K Ludloff (1908) Zur blutigen Einrenkung der angeborenen Huftluxation. Z Orthop Chir 22: 272-276.

3. Kiely N, Younis U, Day JB, et al. (2004) The ferguson medial approach for open reduction of developmental dysplasia of the hip. A clinical and radiological review of 49 hips. J Bone Joint Surg $\mathrm{Br} 86:$ 430-433.

4. Koizumi W, Moriya H, Tsuchiya K, et al. (1996) Ludloff's medial approach for open reduction of congenital dislocation of the hip. A 20-year follow-up. J Bone Joint Surg Br 78: 924-929.

5. P Chiron, J Murgier, E Cavaignac, et al. (2014) Minimally invasive medial hip approach. Orthop Traumatol Surg Res 100: 687-689.

6. W Thomas, P Benecke (2004) Der mediale Zugang zum Hüftgelenk zur Implantation von Endoprothesen. Operative Orthopädie und Traumatologie 16: 288-299.

7. W Thomas, L Lucente, P Benecke (2007) The medial approach to the hip joint for implantation of prostheses. In: Stiehl JB, Konermann WH, Haaker RG, Navigation and MIS in Orthopedic Surgery. Springer, Berlin, Heidelberg.

8. W Thomas, L Lucente, N Mantegna, et al. (2005) Approccio mediale all'anca per l'impianto di artroprotesi. GIOT 31: 26-29.

9. Honl M, Schwieger K, Morlock MM, et al. (2006) The medial approach in primary total hip replacement surgery, a microinvasive single incision technique in a prospective study. 52nd Annual Meeting of the Orthopaedic Research Society, 0449. 